\title{
EXTRACCIÓN DE COMPUESTOS CON ACTIVIDAD ANTIOXIDANTE DE FRUTOS DE GUAYABA CULTIVADA EN VÉLEZ-SANTANDER, COLOMBIA
}

\section{Diana-Chavely Restrepo-Sánchez, Carlos-Eduardo Narváez-Cuenca* y Luz-Patricia Restrepo-Sánchez}

Departamento de Química, Facultad de Ciencias, Universidad Nacional de Colombia, Ciudad Universitaria, Bogotá-Colombia. Cra $30 \mathrm{~N}^{\circ} 45-03$

Recebido em 8/8/08; aceito em 28/1/09; publicado na web em 3/7/09

\begin{abstract}
EXTRACTION OF COMPOUNDS WITH ANTIOXIDANT ACTIVITY IN GUAVA (Psidium guajava L.) FRUIT PRODUCED IN VÉLEZ-SANTANDER, COLOMBIA. The effects of the extraction system (50\% methanol or 50\% methanol pH 2.0), volume/ material ratio, temperature, time and extractions with $70 \%$ acetone were evaluated in the total phenolic compounds (TPC) extraction and in antioxidant activities (AA) using FRAP and ABTS assays in guava fruit. The best yield was obtained when $0.5 \mathrm{~g}$ of guava were extracted first with $20 \mathrm{~mL} \mathrm{50 \%} \mathrm{methanol} \mathrm{and} \mathrm{then} \mathrm{four} \mathrm{times} \mathrm{with} 20 \mathrm{~mL} 70 \%$ acetone during $30 \mathrm{~min}$ at $50{ }^{\circ} \mathrm{C}$. Among the different trials guava fruit exhibited high levels of AA as well as TPC.
\end{abstract}

Keywords: FRAP; ABTS; total phenolic compounds.

\section{INTRODUCCIÓN}

En Colombia la guayaba ocupa un tercer lugar en importancia en la comercialización de frutas, después de los cítricos y el banano, con una producción anual alrededor de 128000 ton. Los principales municipios productores de guayaba del país son Vélez, Guavatá, Jesús María, Barbosa y Puente Nacional, todos ubicados en el Departamento de Santander (Figura 1). En el municipio de Vélez se produce cerca del $82 \%$ de la producción nacional. La guayaba se utiliza como materia prima para la elaboración de bocadillo, néctar, sirope, para la obtención de pulpas o para el consumo directo como fruto fresco. Aunque se cultivan diferentes variedades, una de las más comercializadas es la guayaba roja. Este fruto tiene un diámetro longitudinal entre 4 a $6 \mathrm{~cm}$ y ecuatorial entre 4 a $5,5 \mathrm{~cm}$, con peso entre 55 a $95 \mathrm{~g} /$ fruta y dos cosechas al año, la principal entre octubre a noviembre $y$, una secundaria, de julio a agosto. En su madurez sensorial tiene una corteza de color amarillo y su pulpa es roja, de sabor dulce.

De la guayaba sobresalen sus agradables características sensoriales y sus características nutricionales. Tiene un aporte importante de fibra ( $49 \%$ base seca), ${ }^{1}$ por lo que posee un suave efecto laxante. Se destacan su alto contenido de vitamina $\mathrm{C} \mathrm{y}$, su aporte en compuestos fenólicos y en su actividad antioxidante. Se reportan valores de vitamina C que oscilan entre 55 a $397 \mathrm{mg} / 100 \mathrm{~g}$ base húmeda (B.H.). ${ }^{1-5}$ El contenido de fenoles totales en diferentes variedades de guayaba oscila entre 148 a 462 mg ácido gálico/100 g B.H., la actividad antioxidante medida por FRAP está entre 15 a $40 \mu \mathrm{mol}$ Trolox/g B.H. y por ABTS se ha reportado un valor de $40 \mu \mathrm{mol}$ Trolox/g B.H., ${ }^{2,4}$

Para la extracción de compuestos fenólicos y medida de actividad antioxidante a partir de guayaba se ha usado la extracción a temperatura ambiente de $0,5 \mathrm{~g}$ de muestras liofilizadas con $20 \mathrm{~mL}$ de metanol:agua $(50: 50 \mathrm{v} / \mathrm{v})$ durante $1 \mathrm{~h}$, seguido de una segunda extracción con $20 \mathrm{~mL}$ de acetona:agua $(70: 30 \mathrm{v} / \mathrm{v})$; las medidas de fenoles y de actividad antioxidante se hacen sobre la mezcla de los extractos. ${ }^{5}$ También se ha propuesto la extracción a temperatura ambiente de $2 \mathrm{~g}$ de guayaba fresca o procesada con $25 \mathrm{~mL}$ de acetona:agua $(50: 50 \mathrm{v} / \mathrm{v})$ durante $1 \mathrm{~h}$ para el análisis de la actividad antioxidante y, la extracción de $2 \mathrm{~g}$ de material con $25 \mathrm{~mL}$ de etanol:agua $(40: 60 \mathrm{v} / \mathrm{v})$ durante $1 \mathrm{~h}$ para los compuestos fenólicos totales. ${ }^{6}$ Existe otro reporte en el que preparan

*e-mail: cenarvaezc@unal.edu.co el extracto de guayaba, para medir fenoles y actividad antioxidante, mediante una primera extracción de $3 \mathrm{~g}$ de material con $25 \mathrm{~mL}$ de metanol y una segunda con $20 \mathrm{~mL}$ de diclorometano; las medidas las efectúan sobre cada fracción de manera separada. ${ }^{4}$ Existen trabajos en otros materiales vegetales en los que se indica que la acidificación del sistema metanol:agua con $\mathrm{HCl}$ puede mejorar la extracción de compuestos fenólicos y la actividad antioxidante. ${ }^{1,7,8}$

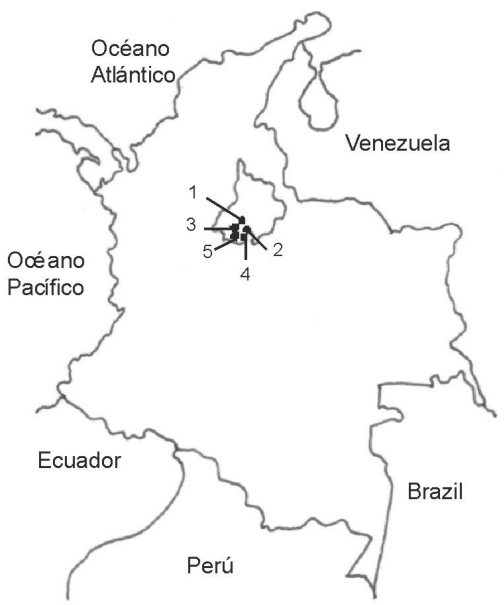

Figura 1. Principales zonas de producción de guayaba roja en Colombia. 1 Vélez, 2 Barbosa, 3 Guavatá, 4 Puente Nacional y 5 Jesús María

La actividad antioxidante puede ser determinada por diferentes métodos, entre los que se encuentra el método ABTS (llamado así por el reactivo 2,2'-azinobis (3-etilbenzotiazolina-6-ácido sulfónico)) y el FRAP (Poder Antioxidante de Reducción Férrica). Dentro de los métodos más rápidos, simples operacionalmente y más reproducibles se encuentran los métodos antes mencionados. El método ABTS consiste en la generación del radical $\mathrm{ABTS}^{+}$, por la reacción entre el ABTS y el persulfato de potasio para producir un cromóforo azul verdoso con absorciones máximas a longitudes de onda de 415, 645, 734 y $815 \mathrm{~nm}$. En presencia de antioxidantes se produce una disminución de la absorbancia del radical $\mathrm{ABTS}^{+}$; los resultados suelen ser expresados como $\mu \mathrm{mol}$ Trolox/g material analizado. Este método puede ser utilizado en 
un amplio rango de $\mathrm{pH}$ y se aplica para sistemas tanto acuosos como orgánicos. De otro lado, el método FRAP consiste en la formación de un complejo férrico con el reactivo TPTZ, el cual en presencia de antioxidantes forma un complejo azul de máxima absorción a 593 nm; los resultados se pueden expresar como $\mu$ mol Trolox/g o como $\mu \mathrm{mol}$ $\mathrm{Fe}(\mathrm{II}) / \mathrm{g}$. Adicionalmente, para estimar la cantidad de fenoles se utiliza el método de Folin Ciocalteu; este método consiste en la reacción entre el reactivo de Folin Ciocalteu de color amarillo y los grupos fenólicos, lo cual produce un complejo de color azul con máxima absorción a 725 $\mathrm{nm}$; los resultados usualmente se expresan como mg ácido gálico/100 g. Se ha encontrado que el método de FRAP y el metodo de FolinCiocalteu muestran alta correlación.9-11

Aunque existen diversos trabajos en donde se reporta tanto la cantidad de compuestos fenólicos totales como la actividad antioxidante en guayaba, no existe un trabajo en el que se exploren diversos factores que pueden influir al momento de efectuar la extracción de este tipo de compuestos contenidos en la fracción comestible de guayaba. Además, no existe información alguna sobre este tipo de medidas en guayaba roja, una de las variedades cultivadas en Colombia. El objetivo de este estudio fue evaluar la incidencia de diversos factores sobre la extracción de compuestos fenólicos con actividad antioxidante a partir de guayaba obtenida del municipio de VélezSantander, principal productor de guayaba en Colombia.

\section{PARTE EXPERIMENTAL}

\section{Material vegetal}

Las muestras de guayaba roja fueron adquiridas en Vélez-Santander (Figura 1), transportadas a Bogotá y analizadas en la Universidad Nacional de Colombia. Las frutas fueron lavadas con agua destilada y desinfectadas por inmersión en hipoclorito de sodio al $1 \%$ por 20 min. Para cada experimento se contó con $1 \mathrm{~kg}$ de frutas maduras, sin congelar, por lo que cada vez que se evaluaba un nuevo efecto se empleó un lote de frutas diferente. Las frutas enteras fueron homogenizadas en un procesador de alimentos, las semillas fueron retiradas y se obtuvo el puré, constituido por la pulpa más la corteza, para efectuar los experimentos. Se evaluaron diferentes condiciones de extracción que incluyeron la composición del sistema de extracción, relación extractante a puré, temperatura de extracción, tiempo de extracción y número de extracciones con acetona. En los extractos obtenidos se midió el contenido de fenoles totales por Folin-Ciocalteu y la actividad antioxidante mediante las técnicas de FRAP y ABTS.

\section{Reactivos}

Los reactivos TPTZ [2, 4, 6-(tri-(2-piridil-s-triazina))] y ABTS (2,2'-azinobis (3-etilbenzotiazolina-6-ácido sulfónico)) fueron adquiridos de Sigma Chemicals Co. (St. Louis, MO, USA). El reactivo Trolox (ácido-6-hidroxi-2, 5, 7, 8 tetrametilcromano-2carboxílico) fue adquirido de Aldrich. El ácido gálico monohidratado $\left(\mathrm{C}_{6} \mathrm{H}_{2}(\mathrm{OH})_{3} \mathrm{COOH} . \mathrm{H}_{2} \mathrm{O}\right)$ de J. T. Baker (Phillipsburg, USA). Los otros reactivos fueron adquiridos de Panreac Química S. A. (Barcelona, España). Todos los reactivos utilizados fueron de grado analítico.

\section{Extracción de compuestos con actividad antioxidante}

Para evaluar el efecto de la composición del sistema de extracción y de la relación puré a extractante se procedió así: se pesó $1,0 \mathrm{~g}$ de puré y se adicionaron 2,0 mL de metanol:agua (50:50, v/v). La mezcla fue puesta en agitación, mediante agitador magnético, durante una hora a temperatura ambiente $\left(20^{\circ} \mathrm{C}\right)$. Posteriormente, se centrifugó a 1.493 $\mathrm{X}$ g durante $10 \mathrm{~min}$ y el sobrenadante se almacenó a $-20^{\circ} \mathrm{C}$. El residuo fue sometido a una segunda extracción con 2,0 $\mathrm{mL}$ de acetona:agua (70:30, v/v) a temperatura ambiente durante $1 \mathrm{~h}$ y se centrifugó por 10 min a $1.493 \mathrm{X} \mathrm{g}$. Los dos sobrenadantes fueron reunidos y almacenados a $-20{ }^{\circ} \mathrm{C}$ en la oscuridad hasta por $24 \mathrm{~h}$ para efectuar los análisis de actividad antioxidante y la cuantificación de compuestos fenólicos. Además de los 2,0 mL de extractante se ensayaron 5,0; 10,0; 20,0 y 30,0 mL de metanol:agua (50:50, v/v), seguido de la segunda extracción con 2,0; 5,0; 10,0;20,0 y 30,0 mL de acetona:agua (70:30, v/v). También se ensayó como extractante 2,0; 5,0; 10,0;20,0 y 30,0 $\mathrm{mL}$ de metanol:agua $(50: 50, \mathrm{v} / \mathrm{v}) \mathrm{pH} 2,0$ y segunda extracción con 2,$0 ; 5,0 ; 10,0 ; 20,0$ y $30,0 \mathrm{~mL}$ acetona acuosa.

El efecto de la temperatura de extracción se evaluó al pesar $0,5 \mathrm{~g}$ de puré y extraer durante $1 \mathrm{~h}$ con 10,0 $\mathrm{mL}$ de metanol:agua (50:50, v/v) a $20{ }^{\circ} \mathrm{C}$, seguido de una segunda extracción durante $1 \mathrm{~h}$ con 10,0 $\mathrm{mL}$ de acetona:agua $(70: 30, \mathrm{v} / \mathrm{v})$ a $20^{\circ} \mathrm{C}$. También se ensayaron ambas extracciones a $50{ }^{\circ} \mathrm{C}$ y otra con metanol a $73{ }^{\circ} \mathrm{C}$ seguida por acetona a $50{ }^{\circ} \mathrm{C}$.

Para evaluar el efecto del tiempo de extracción se pesaron muestras de 0,5 g y se extrajeron con 10,0 mL de metanol:agua (50:50, v/v) durante $30,60,120$ y $240 \mathrm{~min}$ a $50{ }^{\circ} \mathrm{C}$ y luego con $10,0 \mathrm{~mL}$ de acetona:agua $(70: 30, \mathrm{v} / \mathrm{v})$ durante $30,60,120$ y $240 \mathrm{~min}$ a $50{ }^{\circ} \mathrm{C}$.

Finalmente, se evaluó el efecto del número de lavados sobre las variables en estudio. Se pesaron muestras de $0,5 \mathrm{~g}$ y se extrajeron con 10,0 mL de metanol:agua $(50: 50, \mathrm{v} / \mathrm{v})$ durante $30 \mathrm{~min}$ a $50{ }^{\circ} \mathrm{C}$. A los residuos obtenidos se les efectuaron extracciones sucesivas, entre una a cinco veces, con $10,0 \mathrm{~mL}$ de acetona acuosa al $70 \%$, cada una, a $50{ }^{\circ} \mathrm{C}$. Se incluyó un tratamiento en el que no se efectuó extracción con acetona.

\section{Fenoles totales}

La cuantificación fue realizada por el método de Folin-Ciocalteu acorde a lo descrito por otros autores. ${ }^{8}$ Se efectuaron curvas de calibración con ácido gálico a concentraciones entre 50 a $500 \mathrm{mg} / \mathrm{mL}$, intervalo en el cual hubo linealidad. Los extractos fueron previamente diluidos en proporciones adecuadas para obtener lecturas de absorbancia dentro de las curvas de calibración (diluciones entre 1 a 10 hasta 4 a 10, volumen de extracto a volumen final). Los resultados se expresaron como mg ácido gálico/100 g puré, B.H.

\section{Determinación de la actividad antioxidante}

Se midió tanto por el método FRAP ${ }^{9}$ como por el método ABTS. ${ }^{11}$ Se efectuaron curvas de calibración con Trolox, para el método FRAP en concentraciones entre 0,1 a 1,5 $\mathrm{mM}$ y para el método ABTS entre 0,25 a 1,5 mM. Se verificó la linealidad de las curvas de calibración en los intervalos. Para obtener lecturas estables de absorbancia, en la medida de actividad de los extractos, fue necesario un tiempo de reacción de 45 min en ABTS y de 2 h en FRAP. Los extractos fueron diluidos en proporciones adecuadas para obtener lecturas dentro de las curvas de calibración (diluciones entre 1 a 20 hasta 4 a 20, volumen de extracto a volumen final).

\section{Análisis de datos}

Los experimentos se efectuaron por triplicado. Cada efecto se evaluó con lotes de fruta diferentes. Sobre las variables de respuesta se determinaron los promedios y las desviaciones estándar. El efecto de la composición del extractante (A) y de la relación puré a volumen de extractante (B) se evaluó en un diseño completamente al azar con arreglo factorial A x B. Los otros experimentos fueron realizados acorde a un diseño completamente al azar. Se realizaron ANOVAS y se aplicó la prueba Tukey para determinar las diferencias 
Tabla 1. Resultados de los ANOVAS efectuados para las variables de respuesta al variar diversos parámetros durante la extracción de compuestos antioxidantes de guayaba

\begin{tabular}{|c|c|c|c|c|c|c|c|}
\hline \multirow{2}{*}{ Fuente de variación } & \multirow{2}{*}{ GL } & \multicolumn{2}{|c|}{ Fenoles } & \multicolumn{2}{|c|}{ FRAP } & \multicolumn{2}{|c|}{ ABTS } \\
\hline & & $\mathrm{CM}$ & $\mathrm{P}$ & $\mathrm{CM}$ & $\mathrm{P}$ & $\mathrm{CM}$ & $\mathrm{P}$ \\
\hline \multicolumn{8}{|c|}{ Efecto del tipo y de la relación volumen de solución de extracción a puré } \\
\hline Extractante & 1 & 5503 & $<0,01$ & 2468 & $<0,01$ & 150 & 0,29 \\
\hline Relación extractante a puré & 5 & 24749 & $<0,01$ & 1017 & $<0,01$ & 73,6 & $<0,01$ \\
\hline Interacción & 5 & 617 & 0,18 & 61,2 & 0,31 & 42,4 & 0,89 \\
\hline Error & 24 & 367 & & 48,1 & & 127 & \\
\hline \multicolumn{8}{|l|}{ Efecto de la Temperatura } \\
\hline Temperatura & 2 & 13158 & $<0,01$ & 2870 & $<0,01$ & 2314 & $<0,01$ \\
\hline Error & 6 & 1112 & & 5,9 & & 111 & \\
\hline \multicolumn{8}{|l|}{ Efecto del tiempo } \\
\hline Tiempo & 3 & 70122 & $<0,01$ & 10140 & $<0,01$ & 2725 & $<0,01$ \\
\hline Error & 8 & 91,4 & & 93,8 & & 50,6 & \\
\hline \multicolumn{8}{|c|}{ Efecto de las extracciones con acetona } \\
\hline Extracciones & 5 & 26380 & $<0,01$ & 5527 & $<0,01$ & 2059 & $<0,01$ \\
\hline Error & 12 & 1827 & & 17,4 & & 232 & \\
\hline
\end{tabular}

GL: grados de libertad; CM: cuadrado medio; P: Probabilidad

entre promedios de tratamientos $(\mathrm{p}<0,05)$. Se calcularon los coeficientes de correlación entre las variables de respuesta por la prueba de Pearson. Los análisis fueron efectuados a través del programa Statgraphics 5,1 plus ${ }^{\circledR}$.

\section{RESULTADOS Y DISCUSIÓN}

La Tabla 1 permite ver que hubo efecto tanto del tipo de extractante como de la relación extractante a puré $(\mathrm{v} / \mathrm{m})$ sobre el contenido de fenoles y sobre la actividad antioxidante medida por la técnica FRAP. Para la técnica ABTS no hubo efecto del tipo de extractante pero sí de la relación extractante a material vegetal. Para ninguna de las tres variables de respuesta hubo efecto de la interacción extractante X relación extractante a puré. Los otros factores, temperatura de extracción, tiempo de extracción y número de extracciones con acetona, tuvieron también un efecto importante sobre las variables estudiadas.
En la Figura 2 se puede apreciar que, en general, el metanol:agua (50:50) arrojó mayores valores de actividad antioxidante medida por FRAP pero, menores valores de fenoles totales que el sistema metanol:agua (50:50) pH 2,0. La respuesta para ABTS fue independiente del tipo de solución de extracción empleada. También se aprecia que al aumentar la relación extractante a puré se incrementaron las respuestas de las variables en estudio. Acorde a la prueba de Tukey, si se emplea una relación 1 a 20 (m/v, puré a extractante) se obtienen resultados máximos ó significativamente iguales a los máximos tanto para la actividad antioxidante medida por FRAP y ABTS como para el contenido de compuestos fenólicos. Cuando la relación metanol:agua (50:50) a puré fue 20 a 1, el contenido de fenoles representó el 160土7\%, si se compara contra el valor obtenido cuando la relación extractante a puré fue 2 a 1. Una comparación similar arroja un valor de $189 \pm 4$ y $131 \pm 29 \%$ para las técnicas de FRAP y ABTS. Aunque con metanol acuoso acidulado el ANOVA indicó que había una mayor respuesta en
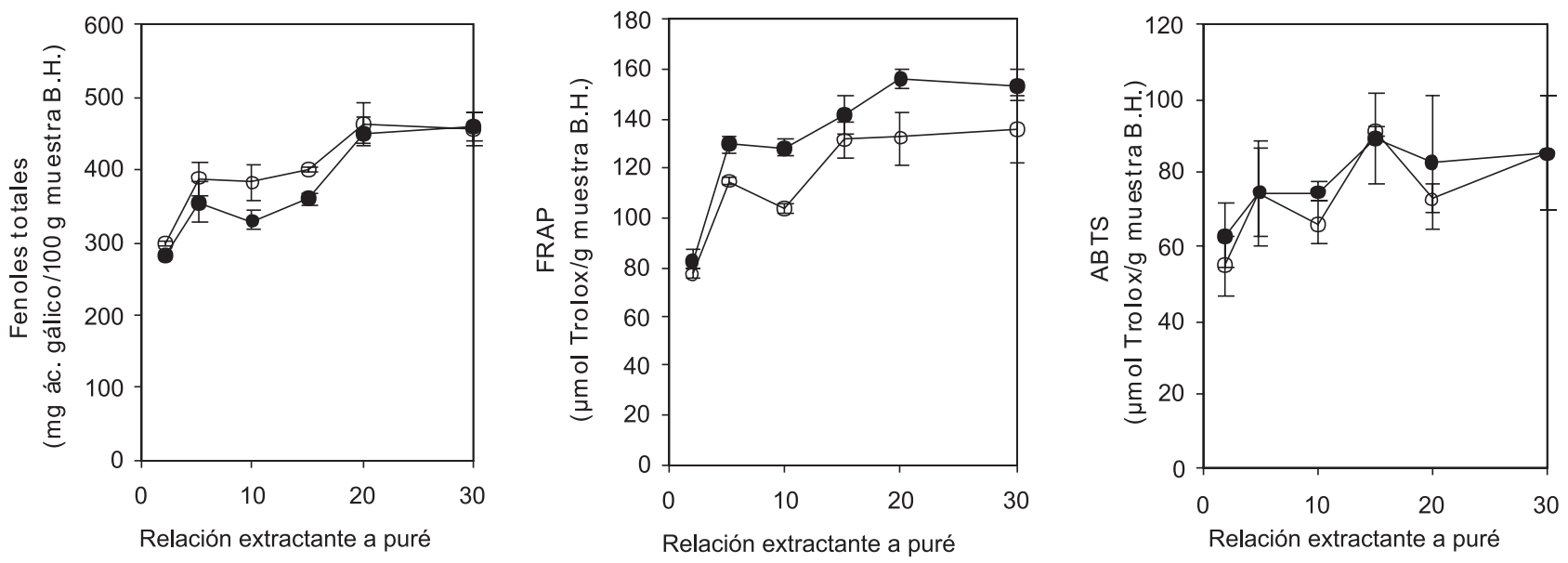

Figura 2. Efecto de la relación extractante a puré y del sistema de extracción sobre la cantidad de fenoles totales y la actividad antioxidante medida por los métodos FRAP y ABTS. Línea continua: metanol acuoso 50\%, seguido de acetona acuosa $70 \%$, línea discontínua: metanol acuoso $50 \%$ pH 2,0, seguido de acetona acuosa 70\%. Los resultados son el promedio \pm 1 desviación estándar ( $n=3)$. Letras diferentes indican diferencias significativas por la prueba de Tukey ( $<<0,05)$ 
los fenoles totales que con metanol acuoso, la prueba de Tukey indicó que no había diferencias significativas entre estos dos tratamientos para un volumen de $20 \mathrm{~mL}$. De acuerdo a las tendencias observadas los siguientes experimentos se efectuaron con el sistema metanol:agua (50:50, v/v) en relación 1 a $20(\mathrm{~m} / \mathrm{v}$, puré a extractante).

$\mathrm{Al}$ evaluar el efecto de la temperatura (Tabla 2) se halló que cuando la temperatura de extracción se subió desde 20 a $50{ }^{\circ} \mathrm{C}$ se presentó un aumento en la cantidad de fenoles extraíbles y en la actividad antioxidante. Para los contenidos de fenoles y actividad antioxidante por FRAP y ABTS, los valores obtenidos a $50{ }^{\circ} \mathrm{C}$ representaron el $145 \pm 8,171 \pm 1$ y $187 \pm 4 \%$ de los obtenidos a $20^{\circ} \mathrm{C}$, respectivamente. Se evidencia que el incremento de la temperatura de extracción desde 20 hasta $50{ }^{\circ} \mathrm{C}$ mejora notablemente la extracción de antioxidantes. A $72{ }^{\circ} \mathrm{C}$ se observó una disminución en la actividad antioxidante y en la cantidad de fenoles, lo cual puede ser un indicativo del efecto de degradación que tiene la temperatura alta sobre los fenoles y otros compuestos con actividad antioxidante.
La inestabilidad de los antioxidantes también se encontró al evaluar el efecto del tiempo de extracción sobre las variables en estudio, ya que al incrementar el tiempo de extracción hubo una disminución en la actividad antioxidante y en la cantidad de fenoles (Tabla 3). Puesto que en los ensayos anteriores se venía trabajando con tiempos de extracción de $1 \mathrm{~h}$, en el ensayo de tiempo de extracción se tomaron los valores obtenidos a 60 min de extracción como el $100 \%$. La disminución desde 1 h hasta 30 min no cambió de manera significativa (Prueba de Tukey) la actividad antioxidante, aunque hubo incrementos que representaron el $108 \pm 3$ y el $103 \pm 3 \%$ respecto a la extracción durante $1 \mathrm{~h}$, para los métodos FRAP y ABTS, respectivamente. Respecto a los compuestos fenólicos hubo un incremento significativo (Prueba de Tukey), que representó el 112 $2 \%$ de lo obtenido cuando las extracciones fueron hechas durante $1 \mathrm{~h}$. Finalmente, se consideró prudente elegir un tiempo de extracción de 30 min, tanto para la extracción con metanol:agua (50:50), como para la extracción con acetona:agua (70:30).

Tabla 2. Efecto de la temperatura de extracción sobre el contenido de fenoles totales y la actividad antioxidante medida por las técnicas de FRAP y ABTS en guayaba

Fenoles (mg AG/100 g muestra B.H.) FRAP ( $\mu$ mol Trolox/g muestra B.H.) ABTS ( $\mu$ mol Trolox/g muestra B.H.)

\begin{tabular}{lcccccc} 
& $\mathrm{M} \pm 1 \mathrm{SD}$ & \%Recuperación & $\mathrm{M} \pm 1 \mathrm{SD}$ & \%Recuperación & M $\pm 1 \mathrm{SD}$ & \%Recuperación \\
\hline $20{ }^{\circ} \mathrm{C}$ & $286 \pm 12 \mathrm{~b}$ & $100 \pm 4$ & $86 \pm 1 \mathrm{c}$ & $100 \pm 1$ & $63 \pm 10 \mathrm{~b}$ & $100 \pm 16$ \\
$50^{\circ} \mathrm{C}$ & $418 \pm 23 \mathrm{a}$ & $145 \pm 8$ & $147 \pm 1 \mathrm{a}$ & $171 \pm 1$ & $118 \pm 2 \mathrm{a}$ & $187 \pm 4$ \\
$73{ }^{\circ} \mathrm{C}$ & $368 \pm 52 \mathrm{ab}$ & $128 \pm 18$ & $108 \pm 4 \mathrm{~b}$ & $125 \pm 5$ & $83 \pm 15 \mathrm{~b}$ & $131 \pm 24$ \\
\hline
\end{tabular}

Cada valor es el promedio \pm 1 desviación estándar $(\mathrm{M} \pm 1 \mathrm{SD}, \mathrm{n}=3)$. Los valores están expresados en base húmeda. Letras diferentes dentro de cada columna indican diferencias significativas por la prueba de Tukey $(\mathrm{p}<0,05)$. Para enlazar con el efecto previo estudiado, tipo de extractante $\mathrm{X}$ volumen de extractante, los valores obtenidos cuando las extracciones se llevaron a cabo a $20{ }^{\circ} \mathrm{C}$ fueron tomados como el $100 \%$.

Tabla 3. Efecto del tiempo de extracción sobre el contenido de fenoles totales y la actividad antioxidante medida por las técnicas de FRAP y ABTS en guayaba

Fenoles (mg AG/100 g muestra B.H.) FRAP ( $\mu$ mol Trolox/g muestra B.H.)

ABTS ( $\mu$ mol Trolox/g muestra B.H.)

\begin{tabular}{|c|c|c|c|c|c|c|}
\hline & $\mathrm{M} \pm 1 \mathrm{SD}$ & \%Recuperación & $\mathrm{M} \pm 1 \mathrm{SD}$ & \%Recuperación & $\mathrm{M} \pm 1 \mathrm{SD}$ & \%Recuperación \\
\hline $30 \mathrm{~min}$ & $626 \pm 11 a$ & $112 \pm 2$ & $275 \pm 7 a$ & $108 \pm 3$ & $164 \pm 4 a$ & $103 \pm 3$ \\
\hline $60 \mathrm{~min}$ & $560 \pm 5 b$ & $100 \pm 1$ & $254 \pm 2 \mathrm{a}$ & $100 \pm 1$ & $159 \pm 10 \mathrm{a}$ & $100 \pm 6$ \\
\hline $120 \mathrm{~min}$ & $338 \pm 8 c$ & $60 \pm 1$ & $193 \pm 8 b$ & $76 \pm 3$ & $119 \pm 8 b$ & $75 \pm 5$ \\
\hline $240 \mathrm{~min}$ & $328 \pm 12 c$ & $59 \pm 2$ & $145 \pm 16 c$ & $58 \pm 6$ & $103 \pm 4 c$ & $65 \pm 2$ \\
\hline
\end{tabular}

Cada valor es el promedio \pm 1 desviación estándar $(\mathrm{M} \pm 1 \mathrm{SD}, \mathrm{n}=3)$. Los valores están expresados en base húmeda. Letras diferentes dentro de cada columna indican diferencias significativas por la prueba de Tukey $(\mathrm{p}<0,05)$. Para enlazar con el efecto previo estudiado, temperatura, los valores obtenidos cuando las extracciones se llevaron a cabo durante 60 min fueron tomados como el $100 \%$.

Tabla 4. Efecto del número de extracciones con acetona sobre el contenido de fenoles totales y la actividad antioxidante medida por las técnicas de FRAP y ABTS en guayaba

\begin{tabular}{|c|c|c|c|c|c|c|}
\hline & \multicolumn{2}{|c|}{ Fenoles (mg AG/100 g muestra B.H.) } & \multicolumn{2}{|c|}{ FRAP ( $\mu$ mol Trolox/g muestra B.H.) } & \multicolumn{2}{|c|}{ ABTS ( $\mu$ mol Trolox/g muestra B.H.) } \\
\hline & $\mathrm{M} \pm 1 \mathrm{SD}$ & \%Recuperación & $\mathrm{M} \pm 1 \mathrm{SD}$ & \%Recuperación & $\mathrm{M} \pm 1 \mathrm{SD}$ & \%Recuperación \\
\hline 0 & $352 \pm 4 c$ & $72 \pm 1$ & $127 \pm 2 d$ & $66 \pm 1$ & $112 \pm 24 b$ & $76 \pm 16$ \\
\hline 1 & $488 \pm 40 b$ & $100 \pm 8$ & $194 \pm 2 c$ & $100 \pm 1$ & $148 \pm 12 \mathrm{ab}$ & $100 \pm 8$ \\
\hline 2 & $532 \pm 37 \mathrm{ab}$ & $109 \pm 8$ & $215 \pm 6 b$ & $111 \pm 3$ & $167 \pm 19 a$ & $113 \pm 12$ \\
\hline 3 & $524 \pm 50 \mathrm{ab}$ & $107 \pm 10$ & $196 \pm 4 c$ & $101 \pm 2$ & $163 \pm 9 a$ & $110 \pm 6$ \\
\hline 4 & $616 \pm 59 a$ & $126 \pm 12$ & $247 \pm 3 a$ & $127 \pm 1$ & $184 \pm 12 \mathrm{a}$ & $124 \pm 8$ \\
\hline 5 & $593 \pm 45 \mathrm{ab}$ & $121 \pm 12$ & $238 \pm 5 a$ & $123 \pm 3$ & $180 \pm 10 \mathrm{a}$ & $122 \pm 7$ \\
\hline
\end{tabular}

Cada valor es el promedio \pm 1 desviación estándar $(\mathrm{M} \pm 1 \mathrm{SD}, \mathrm{n}=3)$. Los valores están expresados en base húmeda. Letras diferentes dentro de cada columna indican diferencias significativas por la prueba de Tukey $(\mathrm{p}<0,05)$. 
Los resultados obtenidos al evaluar el efecto del número de extracciones con acetona se muestran en la Tabla 4. Se observó que tanto la actividad antioxidante, como la cantidad total de fenoles tendieron a aumentar a medida que se aumentaron los lavados con acetona:agua (70:30). Puesto que en los ensayos anteriores se venía haciendo una extracción con metanol acuoso y otra con acetona acuosa se tomó ese tratamiento como el $100 \%$. El contenido de fenoles, al efectuar la extracción con metanol acuoso, sin extracción posterior con acetona, representó un $72 \%$ del valor obtenido en la extracción con metanol:agua $(50: 50 \mathrm{v} / \mathrm{v})$ y luego con acetona:agua $(70: 30 \mathrm{v} / \mathrm{v})$. Al hacer una comparación similar para la actividad antioxidante medida por FRAP y ABTS se encontró que representaban el $66 \pm 1$ y $76 \pm 16 \%$, respectivamente. El porcentaje de recuperación de actividad antioxidante y de fenoles se hizo máximo luego de 4 extracciones con acetona:agua (70:30) y, respecto al tratamiento en el que se efectuó una extracción con acetona, se obtuvieron valores de fenoles y de actividad antioxidante por FRAP y ABTS que representaron el $126 \pm 12,127 \pm 1$ y $124 \pm 8 \%$, respectivamente.

Durante los diferentes experimentos se evidenció un efecto importante tanto de las variables independientes estudiadas como también del lote de frutas estudiado sobre el contenido de compuestos fenólicos y la actividad antioxidante. Al evaluar la mejora en la extracción, desde las respuestas más bajas obtenidas en el primer ensayo: $1 \mathrm{~g}$ de puré extraído con 2,0 mL de metanol:agua (50:50) durante $1 \mathrm{~h}$ a 20 ${ }^{\circ} \mathrm{C}$ seguido por 2,0 $\mathrm{mL}$ de acetona:agua (70:30) durante $1 \mathrm{~h} \mathrm{a} 20{ }^{\circ} \mathrm{C}$, hasta las respuestas más altas, obtenidas en el último: $0,5 \mathrm{~g}$ de puré extraído con 10,0 mL de metanol:agua (50:50) durante 30 min a 50 ${ }^{\circ} \mathrm{C}$ seguido por cuatro extracciones con volumenes de $10,0 \mathrm{~mL}$, cada una, de acetona:agua (70:30) durante $30 \mathrm{~min}$ a $50{ }^{\circ} \mathrm{C}$, se obtuvieron valores que representaron el 327,443 y $381 \%$, para los fenoles totales y la actividad antioxidante por FRAP y ABTS, respectivamente.

Los coeficientes de correlación de Pearson evidenciaron que existe correlación $(p<0,01)$ entre las tres variables de respuesta en estudio (Figura 3). Correlaciones entre estas variables han sido reportadas previamente. ${ }^{4,5,12} \mathrm{El}$ valor para la relación $\mathrm{ABTS}^{+} /$/FRAP durante los diferentes experimentos varió entre 0,38 a 0,88 . Otros autores han reportado intervalos un poco más amplios cuando evalúan la misma relación para diferentes frutas y hortalizas (entre 0,6 a $2,5^{5}$ y entre 0,7 a $3,3^{12}$ ).

Los valores de las pendientes para la relación FRAP/fenoles y $\mathrm{ABTS}^{+}+$ffenoles halladas en este trabajo fueron 0,40 y 0,34 , respectivamente (Figura 3), lo que sugiere que los fenoles presentes en la guayaba generan una respuesta un poco mayor ante la técnica de FRAP que para ABTS. Esta misma conclusión se puede sacar al evaluar la pendiente de la relación ABTS ${ }^{+} /$FRAP, en la que se obtiene un valor de 0,60 . Se ha indicado que ambas técnicas, ABTS y FRAP, son adecuadas para medir la actividad antioxidante de frutas y hortalizas y que los antioxidantes contenidos en este tipo de materiales tienen diferente reactividad frente a los dos métodos. ${ }^{12}$ Aunque ambas técnicas, ABTS y FRAP basan su método en reacciones de tipo redox, con potencial redox similares, las diferencias en las respuestas ante las dos técnicas pueden radicar en el pH de trabajo, en ABTS se trabaja a $\mathrm{pH}$ neutro en tanto que en FRAP se trabaja a $\mathrm{pH}$ 3,6 por lo que se disminuye el potencial de ionización involucrado en la transferencia de electrones, con lo que se incrementa el potencial redox y finalmente se cambia el mecanismo de reacción dominante. Además, el mecanismo de reacción en FRAP es por transferencia de electrones en tanto que en ABTS es tanto por transferencia de electrones como por transferencia de átomos de $\mathrm{H}^{10}$

Durante las diferentes pruebas realizadas se encontró que el contenido de fenoles estuvo en un rango entre 286 hasta $626 \mathrm{mg}$ ácido gálico/100 g muestra B.H., intervalo que cubre el valor reportado para guayaba en un trabajo previo $(462 \pm 128 \mathrm{mg}$ ácido gálico/100 g muestra B.H.), ${ }^{5}$ pero superior a otro reporte $(131 \pm 25$ $\mathrm{mg}$ ácido gálico/100 g muestra B.H.). ${ }^{6} \mathrm{La}$ actividad antioxidante medida por FRAP estuvo entre 86 a $275 \mu$ mol Trolox/g muestra B.H., intervalo superior a los valores reportados previamente por otros autores, $45 \pm 14 \mu \mathrm{mol}$ Trolox/g muestra B.H., ${ }^{5} 8,1 \pm 1,7 \mu \mathrm{mol}$ Trolox/g muestra B.H. ${ }^{6}$ y, 43,6 y 73,6 $\mu$ mol Trolox/g muestra B.H. para pulpa y corteza de guayaba. ${ }^{13}$ Estos dos últimos valores están calculados a partir de los reportados por ellos (60,7 y 102,4 $\mu \mathrm{mol}$ $\mathrm{Fe}^{2+} / \mathrm{g}$ muestra B.H.), para lo cual se hicieron curvas de calibración tanto con $\mathrm{Fe}$ (II) como con Trolox. La actividad antioxidante medida por la técnica de ABTS para guayaba se ubicó entre 55 a $184 \mu \mathrm{mol}$ Trolox/g muestra, intervalo superior al reportado para este tipo de fruta en un trabajo previo $(46 \pm 11 \mu \mathrm{mol}$ Trolox $/ \mathrm{g}$ muestra B.H.). ${ }^{5}$

\section{CONCLUSIONES}

Durante la planeación del experimento se consideró que era mejor evaluar el contenido de fenoles y la actividad antioxidante en los frutos maduros frescos, sin someterlos a congelación, con el propósito de evitar posibles cambios que pudieran presentarse durante el almacenamiento congelado. Sin embargo, al emplear lotes diferentes de frutas para evaluar el efecto de los diferentes factores se generó una nueva fuente de variación. A pesar de esto fue posible evidenciar la mejora en la extracción con los diferentes tratamientos ensayados. Dentro de estos tratamientos, con los que se obtuvo mayor aumento en la extracción fueron la relación puré a volumen de extractante y la temperatura de extracción. El procedimiento con el que se obtuvo tanto la máxima extracción de fenoles como los mayores valores en las capacidades antioxidantes es aquel en el que se hace una primera extracción con metanol acuoso $50 \%$ a una
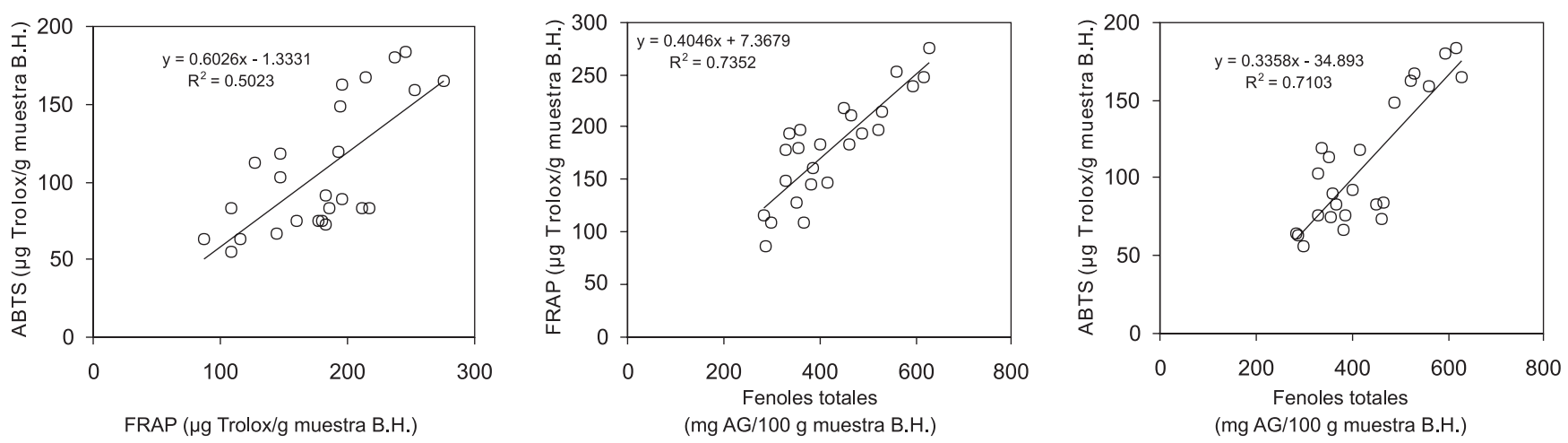

Figura 3. Correlaciones entre las variables de respuesta fenoles totales, ABTS y FRAP. En los tres casos p<0,01 
relación $20 \mathrm{~mL}$ a $1 \mathrm{~g}$ (volumen de extractante a masa de material) a $50{ }^{\circ} \mathrm{C}$ durante $30 \mathrm{~min}$ y cuatro extracciones sucesivas con acetona acuosa $70 \%$ a una relación $20 \mathrm{~mL}$ a $1 \mathrm{~g}$ (volumen de extractante a masa de material) a $50{ }^{\circ} \mathrm{C}$ durante $30 \mathrm{~min}$. Los valores de actividad antioxidante obtenidos en el presente estudio ponen a la guayaba roja cultivada en la región de Vélez-Santander como una buena fuente de antioxidantes.

\section{AGRADECIMIENTOS}

Los autores agradecen el apoyo económico brindado por el Ministerio de Agricultura y Desarrollo Rural de Colombia, a través del proyecto "Obtención de antioxidantes y fibra dietaria a partir de la guayaba" del programa de investigación "Desarrollo de productos funcionales promisorios a partir de la guayaba para el fortalecimiento de la cadena productiva". También se desea agradecer a la Universidad Nacional de Colombia.

\section{REFERENCIAS}

1. Jimenez-Escrig, A.; Rincon, M.; Pulido, R.; Saura-Calixto, F.; J. Agric. Food Chem. 2001, 49, 5489.

2. Bashir, H. A.; Abu-Bark, A.; Abu-Goukh, A. A.-B.; Food Chem. 2003, $80,557$.

3. Mercado-Silva, E.; Benito-Bautista, P.; Garcia-Velasco, M. A.; Postharvest Biol. Technol. 1998, 13, 143.

4. Thaipong, K.; Boonprakob U.; Crosby, K.; Cisneros-Zevallos, L.; Hawkins, D.; J. Food Compos. Anal. 2006, 19, 669.

5. Vasco, C.; Ruales, J.; Kamal-Eldin, A.; Food Chem. 2008, 111, 816.

6. Patthamakanokporn, O.; Puwastien, P.; Nitithamyong, A.; Sirichakwal, P. P.; J. Food Compos. Anal. 2008, 21, 241.

7. Narváez, C. C. E.; Revista Colombiana de Química 2003, 32, 93.

8. Velioglu, Y. S.; Mazza, G.; Gao, L.; Oomah, B. D.; J. Agric. Food Chem. 1998, 46, 4113 .

9. Benzie, F. F.; Strain, J. J.; Anal. Biochem. 1996, 239, 70.

10. Prior, R.; Wu, X.; Schaich. K.; J. Agric. Food Chem. 2005, 53, 4290.

11. Re, R.; Pellegrini, N.; Proteggente, A.; Pannala, A.; Yang, M.; RiceEvans, C.; Free Radical Biol. Med. 1999, 26, 1231.

12. Nilsson, J.; Pillai, D.; Onning, G.; Persson, C.; Nilsson, A.; Akesson, B.; Mol. Nutr. Food Res. 2005, 49, 239.

13. Guo, Ch.; Yang, J.; Wei, J.; Li, Y.; Xu, J.; Jiang, Y.; Nutr. Res. 2003, 23 , 1719. 